\title{
Produção e avaliação de material didático alternativo para o ensino de Neuroanatomia
}

\author{
Mônica Marins \\ Doutora em Ciências Morfológicas pela Universidade Federal do Rio de Janeiro (UFRJ) \\ Professora associada do Laboratório ACESIN, Letras, UFRJ \\ Marilene Gloria da Silva \\ Graduada pelo curso de Fisioterapia da Universidade Veiga de Almeida \\ Daniella Karim Ferreira \\ Graduanda do curso de Fonoaudiologia da Universidade Veiga de Almeida
}

\section{Débora Baltazar dos Santos}

Fonoaudióloga pela Universidade Veiga de Almeida. Pós-graduanda em Musicoterapia pelo Conservatório Brasileiro de Música (CBM)

\section{Danielle Paes-Branco}

Doutora em Fisiopatologia Clínica e Experimental pela Universidade Estadual do Rio de Janeiro (UERJ). Professora da Universidade Veiga de Almeida e Pós-Doutoranda do Laboratório de Neurofisiologia, Departamento de Ciências Fisiológicas, UERJ

$\triangle$ dani_pmab@yahoo.com.br

Recebido em 11 de maio de 2020
Aceito em 23 de março de 2021

\section{Resumo:}

O ensino da Neuroanatomia é um grande desafio, uma vez que a nomenclatura não é de uso cotidiano e a visualização de estruturas que compõem esse sistema pode dificultar o entendimento. Diante das dificuldades, cabe ao professor estimular os discentes com alternativas que possam facilitar o entendimento do conteúdo. Algumas alternativas têm se mostrado eficazes no que diz respeito ao maior envolvimento dos alunos durante a disciplina com ganho considerável no aprendizado. Neste artigo elaboramos um material didático alternativo para o ensino do conteúdo sobre Medula Espinal, na disciplina de Neuroanatomia. Foram selecionadas para reprodução em EVA, imagens ampliadas de cortes de três níveis medulares. Cada corte continha regiões de interesse para encaixe (substância branca, substância cinzenta, vias sensoriais e motoras). Após aprovação pelo Comitê de ética em pesquisa, a atividade foi feita com os alunos divididos em grupos, cada grupo recebeu um conjunto de três cortes (com suas partes desmontadas) e uma lista de perguntas sobre o tema (para nota da disciplina). Após as atividades em sala de aula os alunos responderam a um questionário anônimo de avaliação com onze perguntas. A análise dos 342 questionários revelou que o método foi aprovado por mais de $94 \%$ dos alunos. Concluímos que o material mostrou-se vantajoso para o processo ensinoaprendizagem por suas dimensões, fácil manuseio e baixo custo.

Palavras-chave: Material didático alternativo; Neuroanatomia; Questionário; Medula espinal. 


\title{
Production and evaluation of alternative didactic material for teaching Neuroanatomy
}

\begin{abstract}
:
The teaching of neuroanatomy is a great challenge, since the nomenclature is not in daily use and the visualization of structures that make up this system makes it difficult to understand. In view of the difficulties, it is up to the teacher to encourage students with alternatives that can facilitate the understanding of the content. Some alternatives have shown to be effective with regard to the greater involvement of students during the discipline with considerable gain in learning. In this article we elaborate an alternative didactic material for the teaching of content about Spinal Cord, in the discipline of Neuroanatomy. Enlarged images of sections of three spinal levels were selected for reproduction in EVA. Each cut contained regions of interest for nesting (white matter, gray matter, sensory and motor pathways). After approval by the Research Ethics Committee, the activity was carried out with students divided into groups, each group received a set of three cuts (with their parts disassembled) and a list of questions on the topic (for the discipline's note). After the classroom activities, students answered an anonymous evaluation questionnaire with eleven questions. The analysis of the 342 questionnaires revealed that the method was approved by more than $94 \%$ of the students. We conclude that the material proved to be advantageous for the teaching-learning process due to its dimensions, easy handling and low cost.
\end{abstract}

Keywords: Alternative teaching material; Neuroanatomy; Questionnaires; Spinal cord.

\section{Producción y evaluación de material didáctico alternativo para la enseñanza de Neuroanatomía}

\section{Resumen:}

La enseñanza de la neuroanatomía es un gran desafío, ya que la nomenclatura no es de uso diario y la visualización de las estructuras que componen este sistema puede dificultar la comprensión. En vista de las dificultades, corresponde al maestro alentar a los estudiantes con alternativas que puedan facilitar la comprensión del contenido. Se ha demostrado que algunas alternativas son efectivas con respecto a la mayor participación de los estudiantes durante la disciplina con una ganancia considerable en el aprendizaje. En este artículo elaboramos un material didáctico alternativo para enseñar el contenido sobre la médula espinal, en la disciplina de la neuroanatomía. Se seleccionaron imágenes ampliadas de secciones de tres niveles medulares para reproducción en EVA. Cada corte contenía regiones de interés para anidar (materia blanca, materia gris, vías sensoriales y motoras). Después de la aprobación del Comité de Ética en Investigación, la actividad se realizó con estudiantes divididos en grupos, cada grupo recibió un conjunto de tres cortes (con sus partes desmontadas) y una lista de preguntas sobre el tema (para la nota de la disciplina). Después de las actividades en el aula, los estudiantes respondieron un cuestionario de evaluación anónimo con once preguntas. El análisis de los 342 cuestionarios reveló que el método fue aprobado por más del $94 \%$ de los estudiantes. Llegamos a la conclusión de que el material demostró ser ventajoso para el proceso de enseñanzaaprendizaje debido a sus dimensiones, fácil manejo y bajo costo.

Palabras clave: Material didáctico alternativo; Neuroanatomía; Cuestionario.

\section{INTRODUÇÃo}

A disciplina de Neuroanatomia é um ramo da Anatomia que estuda as estruturas do sistema nervoso e está presente como componente curricular de grande parte dos cursos da saúde e de Psicologia. Além disso, também é uma das diferentes abordagens das neurociências 
para o estudo do sistema nervoso (LENT, 2010). É considerada uma ciência básica e geralmente lecionada nos períodos iniciais destes cursos. O objetivo desta disciplina é compreender e relacionar o nome a cada região específica do sistema nervoso humano. Assim, a Neuroanatomia irá servir de base para disciplinas avançadas de todos os cursos da área da saúde como: Fisiologia humana, Neuropsicologia, Psicologia cognitiva, Psicofarmacologia, Fisioterapia neurológica, entre outras. Portanto, o bom entendimento da matéria ministrada é de grande importância para a vida profissional dos discentes.

O processo de ensino-aprendizagem se apresenta complicado e difícil no que diz respeito ao ensino em morfologia, visto que a memorização da grande quantidade de estruturas e seus nomes complexos torna a tarefa monótona e desestimulante para a maior parte dos alunos, quando não ministrada de maneira mais participativa. O modo como o professor aborda o conteúdo pode repercutir positiva ou negativamente no processo de ensino-aprendizagem do educando (RAMOS et al., 2008). O ensino da Neuroanatomia vem sendo visto como um grande desafio, uma vez que a nomenclatura não é de uso cotidiano e a visualização de estruturas que compõem esse sistema dificulta o entendimento para a maioria dos discentes. Diante dessas dificuldades, cabe ao professor estimular os discentes com alternativas que possam facilitar o entendimento do conteúdo. Os professores e demais profissionais da universidade podem trazer, para o cotidiano estudantil e para a própria sala de aula, práticas que despertem o interesse dos alunos, que tornem a permanência na universidade mais prazerosa e harmônica (PAULA, 2012).

Algumas alternativas vêm apresentando resultados e têm se mostrado eficazes no que diz respeito ao maior envolvimento dos alunos durante a disciplina e que resultam em maior aprendizado (AVERSI-FERREIRA et al., 2008; DE FARIA et al., 2016; SILVA et al., 2017). Alguns autores reconhecem a importância de se criar alternativas que se afastem do quadro e giz e da apresentação de imagens em slide, para que haja maior envolvimento dos alunos e também melhor assimilação e entendimento do conteúdo ministrado (SILVA et al., 2011; SILVA; MACHADO; BIAZUSSI, 2012). Dessa forma, o educador precisa atuar de maneira eficaz, com didáticas inovadoras e necessita possuir competência que vai além do domínio dos conteúdos da disciplina, passando pelo conhecimento de propostas alternativas, para exigir mais do aluno na disciplina e não apenas o exercício de sua capacidade de memorização das estruturas 
anatômicas, mas o conhecimento de sua correlação com as ciências morfológicas e com a prática do curso (CAMPUS NETO; MAIA; GUERRA, 2008).

A diversidade de alunos que possuímos em nossas salas de aula resulta em diferentes níveis de elaboração cognitiva. Enquanto alguns alunos são hábeis em lidar com o conteúdo, outros apresentam sérias dificuldades para compreender conceitos básicos e, portanto, não conseguem visualizar as estruturas que compõem o Sistema Nervoso, não acompanham o raciocínio das explicações e muitas vezes fazem inferências que os levam a conclusões incorretas acerca do assunto lecionado (CARVALHO, 2010). Uma vez que as unidades componentes do sistema forem conhecidas e simbolicamente manipuladas pelos alunos poderá ser possível que eles consigam construir uma memória concreta para que posteriormente possam imaginar as correlações possíveis sobre o funcionamento do sistema ou mesmo que tipo de dano uma lesão poderia causar. Assim, cria-se a possibilidade de guiar o raciocínio dos alunos de modo a sedimentar melhor o conhecimento, proporcionar qualidade na aprendizagem e produzir um reflexo nas avaliações quantitativas do semestre.

Apesar da existência de várias fontes, (livros, artigos científicos e teses) que podem ser utilizadas para estudar a Neuroanatomia, há uma grande busca pela produção de novos métodos que venham facilitar o processo de ensino e aprendizagem (DE FARIA et al., 2016; SILVA et al., 2017). Portanto, torna-se imprescindível a criação de novas alternativas promovendo aos alunos maior interação e assimilação do conteúdo (SHOLL-FRANCO; VEQUE, 2013). Dessa forma o presente estudo envolveu a produção de material didático alternativo para a disciplina de Neuroanatomia visando aumentar o interesse pela disciplina, facilitar o entendimento das aulas teóricas e buscar melhores resultados no aproveitamento do conteúdo para o curso desenvolvido. O uso dos materiais foi avaliado qualitativamente por meio da aplicação de questionários ao final da atividade e pode se tornar uma alternativa interessante para a as aulas teóricas e práticas.

\section{METODOLOGIA}

O estudo foi desenvolvido nas turmas da disciplina de Neuroanatomia de uma universidade particular do Rio de Janeiro. A disciplina é ofertada para os cursos da área da 
saúde sendo obrigatória para os cursos de Psicologia, Fonoaudiologia e Fisioterapia. Em reunião do grupo de pesquisa (composto por duas professoras e três alunos de graduação) ficou decidido que o tópico da ementa de Neuroanatomia escolhido para os modelos seria a Medula Espinal e suas vias. A escolha se justifica pela complexidade do assunto e dificuldade reportada comumente pelos estudantes.

Utilizou-se material de baixo custo para a confecção dos modelos, como: folhas de material emborrachado (EVA) colorido, cola própria para EVA, tesouras, papel cartão, cola branca e cópias de imagens (Figura 1) de dois livros de Neuroanatomia (MACHADO; HAERTEL, 2014; HANSEN, 2015) como base para a confecção do material.

Figura 1. Imagens escaneadas dos cortes medulares e vias nervosas selecionados para produção em EVA.

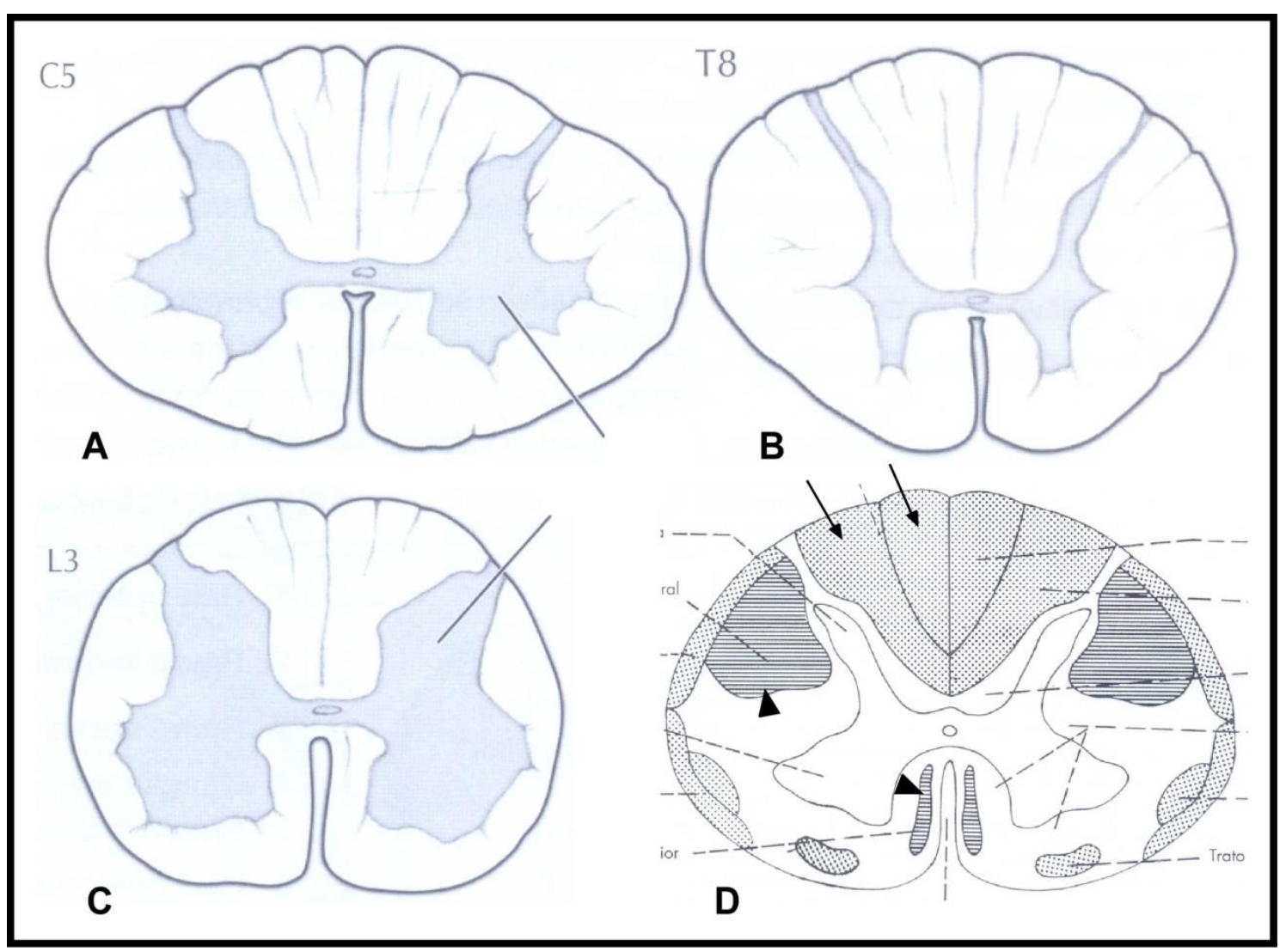

Legenda: Foram selecionados um corte do nível cervical (A), um do toráxico (B) e um do lombar (C). As vias nervosas escolhidas para serem representadas foram os fascículos Grácil e Cuneiforme, das vias ascendentes (D, setas) e os fascículos lateral e medial, das vias descendentes (D, cabeças de seta).

Fonte: MACHADO; HAERTEL, 2014; HANSEN, 2015. 
Para a confecção, o grupo de pesquisa se reunia no Laboratório de Biologia da instituição. Com base no estudo das estruturas internas da medula espinal, bem como nas vias nervosas que por ela trafegam, as imagens de cortes medulares de três níveis diferentes (lombar, torácico e cervical) foram selecionadas para ampliação e impressão em papel A4. Depois de produzidas as cópias, foram selecionadas as estruturas a serem reproduzidas em EVA: substância branca e cinzenta, vias descendentes lateral e medial (vias motoras) e os fascículos Grácil e Cuneiforme, ambos das vias ascendentes (vias sensoriais). As imagens foram transpostas para o papel cartão e recortadas para servirem de molde para riscar o EVA, que foi recortado manualmente em seguida. Cada corte da medula espinal em EVA foi formado por 3 fatias idênticas superpostas e coladas, sendo que duas delas eram vazadas para receber o encaixe das estruturas a serem estudadas. As estruturas diferentes foram recortadas em EVA com cores diferentes, montadas e coladas aos pares, para posterior sobreposição (Figura 2). Os cortes medulares, dos três níveis selecionados com suas respectivas estruturas internas, formaram um conjunto para ser manuseado pelos alunos.

Após a produção da totalidade das peças anatômicas, a atividade foi realizada com os alunos em sala de aula juntamente com a explicação do conteúdo. Cada grupo de alunos era composto por em média de 3 a 6 participantes, dependendo do tamanho da turma. Após a divisão dos grupos, cada um recebia uma pasta contendo as peças de medula e suas respectivas estruturas para serem montadas como parte da atividade.

O projeto foi enviado para apreciação do Comitê de Ética em Pesquisa da Universidade Veiga de Almeida e foi aprovado sob número: 2.701.605. Após a atividade, um questionário, sem a identificação do respondente, foi ministrado para que os discentes avaliassem a atividade bem como a inovação da técnica proposta para o aprendizado. o questionário para avaliação do método de ensino foi elaborado com base na literatura, composto por 11 questões de múltipla escolha e com a possibilidade de justificativa em apenas uma delas. Juntamente com o questionário, o aluno recebeu e assinou um termo de consentimento livre e esclarecido se mostrando de acordo com o uso de suas respostas para pesquisa. 
Figura 2. Cortes da medula espinhal produzidos em EVA.

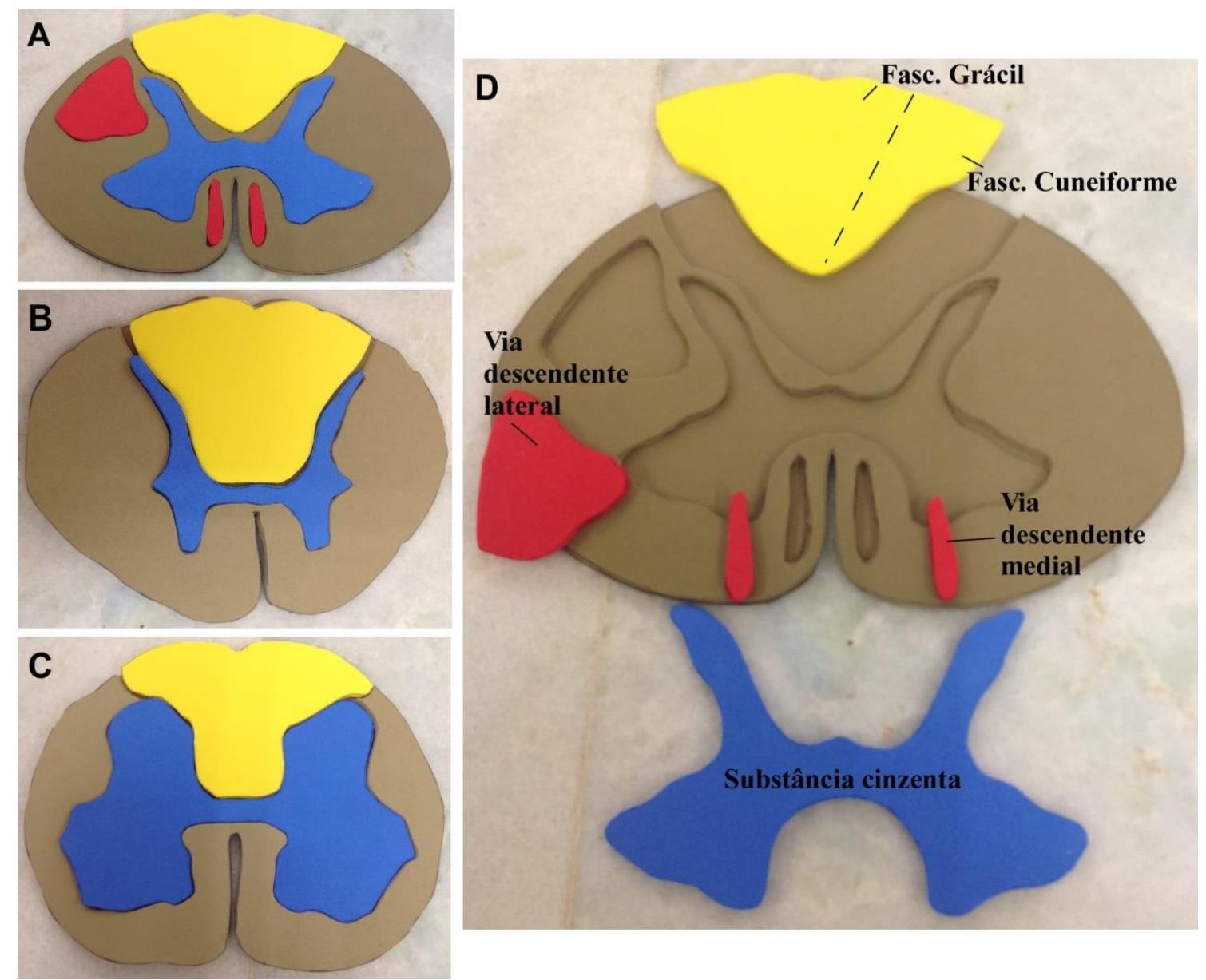

Legenda: As vias ascendentes foram produzidas em EVA amarelo, as descendentes em vermelho, a substância cinzenta em azul e a substância branca (base do corte) em marrom. A substância branca foi composta por 3 fatias idênticas de EVA, sendo apenas duas vazadas, todas superpostas e coladas uma a uma. As demais estruturas foram constituídas de dois recortes idênticos de EVA colados. Após o encaixe das peças todas ficavam no mesmo nível. A. Corte do nível cervical. B. Corte do nível toráxico. C. Corte do nível lombar. D. Corte do nível cervical com suas peças identificadas e retiradas do encaixe.

Fonte: acervo dos autores.

\section{RESULTADOS E DISCUSSÃo}

O questionário foi aplicado em 350 alunos, mas 8 foram excluídos por terem sido entregues em branco. Dessa forma, procedeu-se a análise de 342 questionários. Desses, 77,8\% estavam cursando os três primeiros períodos da faculdade, enquanto $22,2 \%$ estavam cursando do quarto até o nono período (questão 3). A maior parte dos alunos que participaram da 
atividade e responderam o questionário é do sexo feminino $(73,1 \%$, questão 4$)$ e está na faixa de 18 à 22 anos (55,6\%, questão 1). Os alunos estão distribuídos entre os três cursos que fazem obrigatoriamente a disciplina e foram convidados a participar da pesquisa (questão 2; Tabela $1)$.

Tabela 1 - Distribuição dos alunos por período, idade, sexo e curso

\begin{tabular}{|c|c|c|c|}
\hline \multicolumn{4}{|c|}{ DISTRIBUIÇÃO DOS ALUNOS } \\
\hline \multicolumn{2}{|c|}{ PERÍODO } & \multicolumn{2}{|c|}{ IDADE } \\
\hline $1^{\circ}$ & $7,3 \%$ & 18 à 22 & $55,6 \%$ \\
\hline $2^{\underline{o}}$ & $25,1 \%$ & 23 à 28 & $27,2 \%$ \\
\hline $3^{\circ}$ & $45,3 \%$ & 29 à 34 & $8,8 \%$ \\
\hline $4^{\mathrm{o}}$ & $8,5 \%$ & 35 à 41 & $3,5 \%$ \\
\hline $5^{\mathrm{o}}$ & $8,5 \%$ & 42 à 47 & $2,6 \%$ \\
\hline $6^{0}$ & $1,5 \%$ & Acima de 48 & $2,3 \%$ \\
\hline $7^{\circ}$ & $0,9 \%$ & \multicolumn{2}{|c|}{ SEXO } \\
\hline $8^{\circ}$ & $0,9 \%$ & Feminino & $73,1 \%$ \\
\hline $9^{\circ}$ & $2,0 \%$ & Masculino & $26,9 \%$ \\
\hline \multicolumn{4}{|c|}{ CURSO } \\
\hline \multicolumn{2}{|c|}{ Fisioterapia } & \multicolumn{2}{|c|}{$30,1 \%$} \\
\hline \multicolumn{2}{|c|}{ Fonoaudiologia } & \multicolumn{2}{|c|}{$50,0 \%$} \\
\hline \multicolumn{2}{|c|}{ Psicologia } & \multicolumn{2}{|c|}{$19,9 \%$} \\
\hline
\end{tabular}

Legenda: Resultado das respostas das questões 1, 2, 3 e 4 do questionário.

Fonte: acervo dos autores.

Quando perguntados sobre qual fonte os alunos usam para estudar (questão 6), 304 responderam que usam o slide do professor. Sabendo que nessa pergunta os alunos poderiam marcar mais de uma opção de resposta, podemos observar na tabela 2 que, os alunos utilizam menos as peças anatômicas (tanto artificiais quanto naturais) como fonte de estudo. A opção "Sites da internet" e a opção "Livro texto" também foram consideravelmente apontadas (sendo 207 e 169, respectivamente). A opção "Outros" foi escolhida por 41 estudantes, sendo que $51 \%$ desses escreveu na descrição de resposta que utiliza as anotações que faz em sala como fonte de estudo. 
Em relação aos fatores que podem prejudicar o aprendizado da disciplina de Neuroanatomia (questão 7), a maior parte dos alunos acredita que a grande dificuldade da disciplina seja a quantidade de nomes que necessitam ser memorizados (Tabela 2). Em segunda opção, os alunos responderam que acreditam que a dificuldade esteja em relacionar estrutura e função.

Tabela 2 - Distribuição de preferências por fontes de estudo e fatores que dificultam o aprendizado.

\begin{tabular}{cc}
\hline FONTES DE ESTUDO & NÚMERO DE SELEÇÕES \\
\hline Livro texto & 169 \\
Livro atlas & 79 \\
Peças anatômicas artificiais & 43 \\
Peças anatômicas naturais & 45 \\
Slides do professor & 304 \\
Sites da internet & 207 \\
Artigos científicos & 72 \\
Outros & 41 \\
\hline FATORES QUE DIFICULTAM O APRENDIZADO \\
\hline Qula expositiva & 259 \\
\hline Dificuldade de relacionar estrutura e & 8 \\
função & 110 \\
Dificuldade de acesso à peças anatômicas & 74 \\
Outro motivo & 22 \\
\hline
\end{tabular}

Legenda: Resultado das respostas das questões 6 e 7 do questionário. Nessas questões o aluno poderia selecionar quantos itens quisesse, sem ordem de preferência.

Fonte: acervo dos autores.

Nosso resultado mostra que a grande dificuldade dos alunos com a disciplina de Neuroanatomia é a quantidade de nomes para memorizar. Segundo Gelb e colaboradores (2002), a localização neuroanatômica é uma habilidade chave no aprendizado da Neurologia que envolve a identificação de possíveis anormalidades no sistema nervoso. Portanto, quanto 
melhor o aprendizado da Neuroanatomia, melhor seria a possibilidade de um diagnóstico correto de uma possível doença neurológica.

O termo "neurofobia" é usado para designar o medo das disciplinas e assuntos ligados à neurociência e foi criado em 1994 por Jozefowicz. O termo foi utilizado pelo autor para descrever médicos e alunos de medicina (JOZEFOWICZ, 1994; SANTOS-LOBATO et al., 2018), mas pode ser ampliado no contexto de nosso estudo se referindo aos alunos das áreas da saúde, como Fisioterapeutas, Fonoaudiólogos e Psicólogos que usualmente encontram em sua prática clínica casos que exigem o conhecimento de Neurociência. Nesse contexto, a criação de estratégias que facilitam o aprendizado, como a criação de modelos, pode otimizar o ensino e motivar os discentes no estudo das disciplinas ligadas à anatomia (AVERSI-FERREIRA et al., 2008; MOTA; MATA; AVERSI-FERREIRA, 2010; SILVA et al., 2017).

A Organização Mundial da Saúde projeta que, até 2030 o número de pacientes que necessitarão de cuidados relacionados às condições neurológicas será de 7\% do total de todas as doenças (AARLI et al., 2006; TAN et al., 2018). Um outro dado relevante é que uma grande quantidade de pacientes com doenças neurológicas vem sendo tratado por profissionais nãoneurologistas que não tem confiança em lidar com essas doenças e acabam gastando um tempo excessivo na busca por material e informações que já deveriam ter sido aprendidas (TAN et al., 2018). Nesse sentido, o entendimento das disciplinas relacionadas à neurociência desde o começo da formação profissional é essencial.

O método foi considerado estimulante (questão 8) por 323 alunos, desses 159 eram do curso de Psicologia, 100 da Fisioterapia e 64 da Fonoaudiologia. Dentro de cada curso o método foi aprovado por 92,98\%, 97,09\% e 94,12\% dos futuros psicólogos, fisioterapeutas e fonoaudiólogos, respectivamente (Tabela 3). A maioria desses alunos (90,09\%; $n=291$ ) considerou-se interessado ou muito interessado por Neuroanatomia (questão 5). Mesmo assim, 298 alunos relataram ter seu interesse pela disciplina aumentado (questão 10) mais ainda. Desses 141 pertenciam ao curso de Psicologia, 96 ao de Fisioterapia e 61 ao de Fonoaudiologia. Dentre aqueles que tiveram seu interesse aumentado 7,38\% ( $\mathrm{n}=22)$ relataram ter nenhum ou pouco interesse pela disciplina contra 92,62\% ( $n=276)$ que relataram ser interessados ou muito interessados. 
Tabela 3 - Respostas para as questões 8 e 10 do questionário

\begin{tabular}{lcc}
\hline $\begin{array}{l}\mathbf{8} \text { - O método foi } \\
\text { estimulante? }\end{array}$ & SIM & NÃO \\
\hline Psicologia & $92,98 \%(\mathrm{n}=159)$ & $7,02 \%(\mathrm{n}=12)$ \\
Fisioterapia & $97,09 \%(\mathrm{n}=100)$ & $2,91 \%(\mathrm{n}=3)$ \\
Fonoaudiologia & $94,12 \%(\mathrm{n}=64)$ & $5,88 \%(\mathrm{n}=4)$ \\
\hline $\mathbf{1 0}$ - Aumentou o interesse? & & \\
\hline Psicologia & $82,46 \%(\mathrm{n}=141)$ & $17,54 \%(\mathrm{n}=30)$ \\
Fisioterapia & $93,2 \%(\mathrm{n}=96)$ & $6,8 \%(\mathrm{n}=7)$ \\
Fonoaudiologia & $89,7 \%(\mathrm{n}=61)$ & $10,3 \%(\mathrm{n}=7)$ \\
\hline
\end{tabular}

Legenda: Resultado das respostas das questões 8 e 10 do questionário, ambas com opção de marcar "sim" ou "não".

Fonte: acervo dos autores.

Por outro lado, 5,55\% $(\mathrm{n}=19)$ dos alunos não considerou o método estimulante, a grande maioria deles pertencia ao curso de Psicologia $(n=12)$ e apresentou pouco interesse $(n=10)$ por Neuroanatomia. De modo surpreendente 5 alunos desse grupo relataram ter seu interesse pela disciplina aumentado, enquanto que a maioria $(n=14)$ referiu o contrário. Resultados semelhantes foram obtidos em outros estudos, que relataram a receptividade dos estudantes a novas metodologias de ensino (FURTADO; GONÇALVES FERREIRA, A. D.; GONÇALVES FERREIRA, A. J., 2013; RUIZ et al., 2014; GREVILLE; DYMOND; NEWTON, 2016; ARANTES; BARBOSA; FERREIRA, 2017). O nível de aprovação das novas metodologias geralmente é tão alto quanto o engajamento dos alunos nas atividades propostas. Além disso, Arantes, Barbosa e Ferreira (2017), que avaliaram uma metodologia de ensino variada, com aulas teóricas e práticas envolvendo diversas fontes de estudo (como exames de imagem, peças anatômicas e casos clínicos), relatam que os estudantes com as melhores notas avaliaram o método melhor do que aqueles com notas mais baixas. Isso indica que o grau de interesse pela disciplina pode influenciar a avaliação feita por cada aluno.

A proposta da atividade em grupo visou promover a interação e a troca de experiência e informações entre os alunos. Quando perguntados sobre a participação do grupo na atividade (questão 9), 84\% dos alunos respondeu que o grupo participou ativamente enquanto 
somente 16\% relatou pouca ou nenhuma participação do grupo (Gráfico 1). Nessa pequena parcela obtivemos o relato de um aluno que disse ter feito o trabalho sozinho.

Gráfico 1 - Percentuais do modo de participação dos grupos na atividade.

\section{Participação dos grupos na atividade}

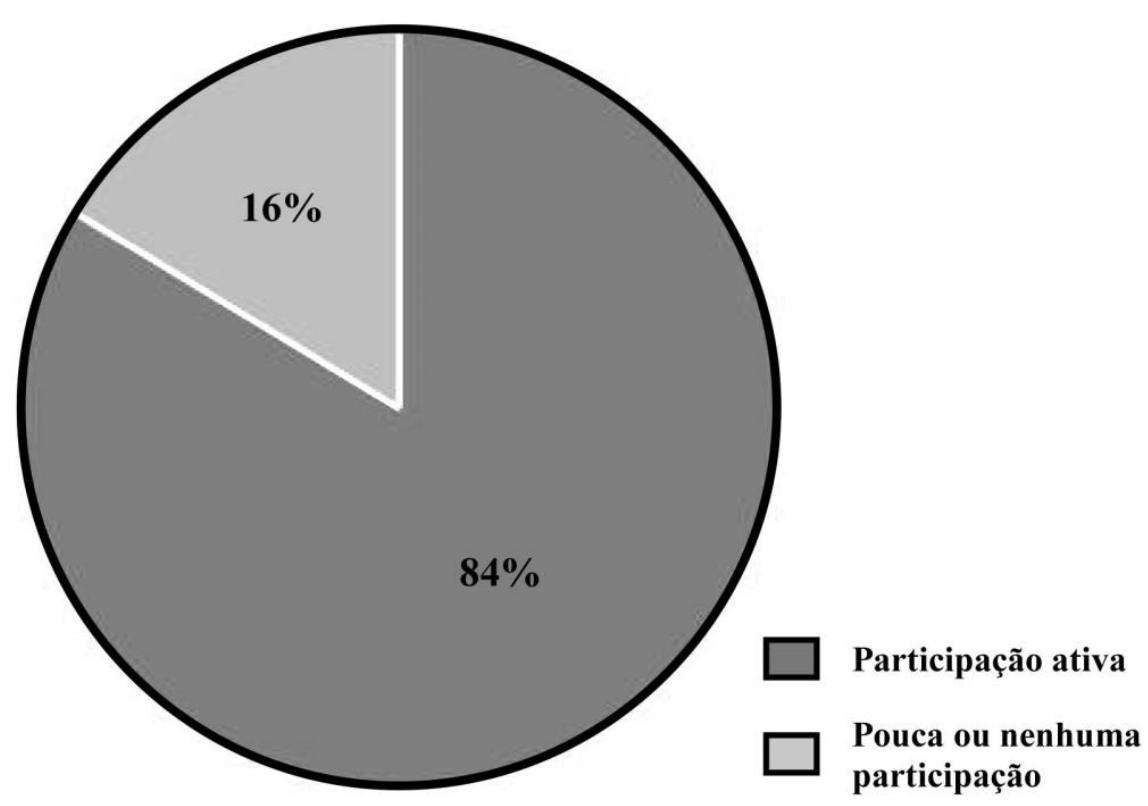

Legenda: Resultados percentuais de respostas para a questão 9.

Fonte: acervo dos autores.

Um paradigma importante da Educação nas últimas décadas têm sido a mudança de um ensino passivo e centrado no professor, para um método ativo e centrado no aluno (SINGH; KHARB, 2013). Os formatos de aprendizagem como palestras e aulas somente teóricas favorecem mais a memorização do que o raciocínio (KRONTIRIS-LITOWITZ, 2008). Nesse contexto, nossa proposta de atividade a ser desenvolvida em grupo, a partir da construção de conhecimento prático, obteve como resultado um relato de participação de $84 \%$ dos alunos perguntados.

A última pergunta do questionário (questão 11) solicitava aos alunos que atribuíssem uma nota para a atividade. As médias das notas atribuídas pelos alunos de cada um dos três cursos ficaram muito próximas e todas acima de 9 (Gráfico 2). Entre os alunos que referiram não ter tido seu interesse por Neuroanatomia aumentado as médias foram ligeiramente 
menores (Fisioterapia = 8,14; Psicologia = 8,27; Fonoaudiologia =8,43), mas como o número de alunos desse grupo era muito pequeno não houve impacto na média final, que ficou muito parecida com as médias dos alunos que referiram ter tido seu interesse pela disciplina aumentado (Fisioterapia =9,4; Psicologia =9,45; Fonoaudiologia =9,56). Nesse sentido, o estudo de Ruiz e colaboradores (2014), que desenvolveu peças neuroanatômicas com menor odor de formalina e com melhor identificação de áreas de interesse, obteve notas dos alunos majoritariamente entre 8,0 e 10. Enquanto que a nota máxima para o aprendizado resultante foi 8,0. Essa diferença parece refletir que as notas atribuídas às peças resultam da satisfação dos alunos ao ter contato com novos materiais de ensino. Embora o presente estudo não tenha medido o grau de aprendizagem dos alunos, as notas por eles atribuídas revelam que sua satisfação foi alcançada.

Gráfico 2 - Notas atribuídas pelos alunos à atividade.

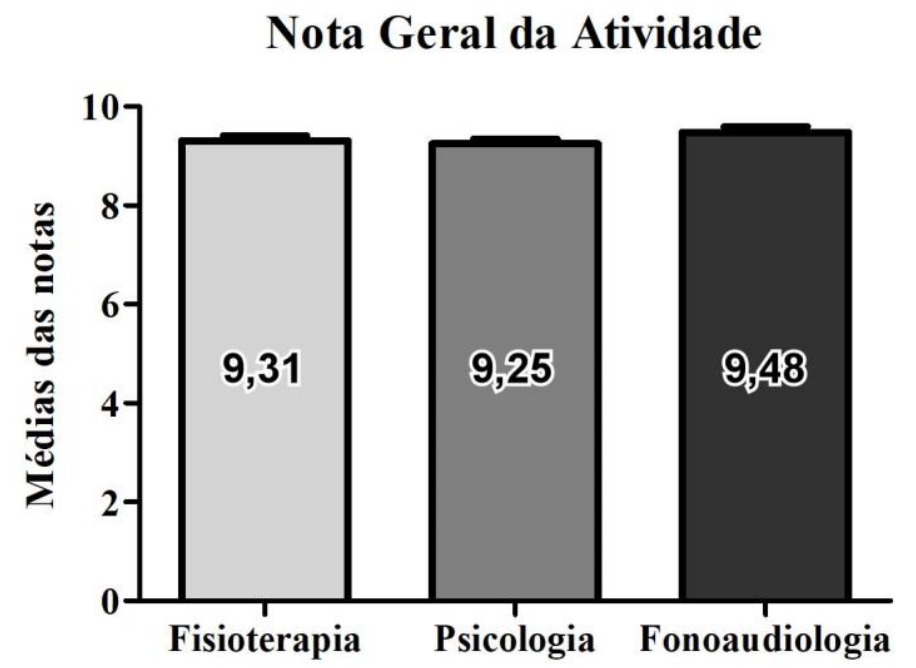

Legenda: As colunas representam as médias das notas atribuídas ao método pelos alunos de cada um dos cursos envolvidos no estudo e em seu interior está o valor numérico das referidas médias. Fonte: acervo dos autores.

\section{CONSIDERAÇÕES FINAIS}

O material desenvolvido possui diversas vantagens, que somarão de modo significativo no desenvolvimento pedagógico dos discentes que farão uso deste. Dentre as vantagens, destacamos o baixo custo, tamanho e forma que facilitam a observação e 
manipulação, favorecendo, deste modo a interação e absorção do conteúdo. Além disso, os questionários mostraram aprovação dos alunos em relação ao novo método.

\section{REFERÊNCIAS}

AARLI, J. A.; DUA, T.; JANCA, A.; MUSCETTA, A. (Editors). Neurological Disorders: Public Health Challenges. Geneva, Switzerland: World Health Organization, 2006. 220 p. Disponível em:

<https://www.who.int/mental_health/neurology/neurological_disorders_report_web.pdf>. Acesso em: 12 out. 2019.

ARANTES, M.; BARBOSA, J.; FERREIRA, M. A. Differences in the students' perceptions on the theaching of neuroanatomy in a medical curriculum organized by disciplines and an integrated medical curriculum. Acta Med. Port., v. 30, p. 1, n. 26-33, 2017. Disponível em:

<https://www.actamedicaportuguesa.com/revista/index.php/amp/article/view/7307>. Acesso em: 28 set. 2019.

AVERSI-FERREIRA, T. A.; MONTEIRO, C. A.; MAIA, F. A.; GUIMARÃES, A. P. R.; CRUZ, M. R. Estudo de neurofisiologia associado com modelos tridimensionais construídos durante o aprendizado. Biosci. J., v. 24, n. 1, p. 98-103, 2008. Disponível em: <http://www.seer.ufu.br/index.php/biosciencejournal/article/view/6750>. Acesso em: 12 mar. 2019.

CAMPUS NETO, F.H.C.; MAIA, N.M.F. e S.; GUERRA, E.M.D. A experiência de ensino da anatomia humana baseada na clínica. Fortaleza: Universidade Metropolitana de fortaleza, Anais do XXIII Congresso Brasileiro de Anatomia, 2008.

CARVALHO, F. A. H. Neurociências e educação: uma articulação necessária na formação docente. Trab. Educ. Saúde, v. 8, n. 3, p. 537-550, 2010. Disponível em: <http://www.scielo.br/scielo.php?pid=S1981-

77462010000300012\&script=sci_abstract\&tlng=pt>. Acesso em: 10 mar. 2019.

DE FARIA, J. W. V.; TEIXEIRA, M. J.; DE MOURA SOUSA JUNIOR, L.; OTOCH, J. P.; FIGUEIREDO, E. G. Virtual and stereoscopic anatomy: when virtual reality meets medical education. Journal of Neurosurgery, v. 125, n. 5, p. 11051111, 2016. Disponível em:

<https://www.researchgate.net/publication/294258392_Virtual_and_stereoscopic_anatomy_When_virtual_reality_ meets_medical_education>. Acesso em: 12 mar. 2019.

FURTADO, I. A.; GONÇALVES FERREIRA, A. D.; GONÇALVES FERREIRA, A. J. Teaching human anatomy to the graduation course in health sciences of the Lisbon university: five years of a new educational experience. Acta Med. Port., v. 26, n. 3, p. 251-257, 2013. Disponível em:

<https://pdfs.semanticscholar.org/da97/b41a1ae5281221c887435add960ae2db27c4.pdf>. Acesso em: 07 set. 2019.

GELB, D. J.; GUNDERSON, C. H.; HENRY, K. A.; KIRSHNER, H. S.; JOZEFOWICZ, R. F.; CONSORTIUM OF NEUROLOGY CLERKSHIP DIRECTORS AND THE UNDERGRADUATE EDUCATION SUBCOMMITTEE OF THE AMERICAN ACADEMY OF NEUROLOGY. The neurology clerkship core curriculum. Neurology, v. 58, n. 6, p. 849-852, 2002. Disponível em: <https://n.neurology.org/content/58/6/849>. Acesso em: 08 out. 2019.

GREVILLE, W. J.; DYMOND, S.; NEWTON, P. M. The student experience of applied equivalence-based instruction for neuroanatomy teaching. J. Educ. Eval. Health Prof., v. 13, p. 32, 2016. Disponível em:

<https://www.semanticscholar.org/paper/The-student-experience-of-applied-equivalence-based-GrevilleDymond/c052dfe20baf9250edd90316897d0bc3f5500f71>. Acesso em: 28 set. 2019.

HANSEN, J. T. Netter anatomia para colorir. 2. ed. Rio de Janeiro: Elsevier, 2015.

JOZEFOWICZ, R. F. Neurophobia: The fear of neurology among medical students. Arch. Neurol., v. 51, n. 4, p. 328329, 1994. Disponível em: <https://jamanetwork.com/journals/jamaneurology/article-abstract/592809>. Acesso em: 08 out. 2019.

KRONTIRIS-LITOWITZ, J. Using truncated lectures, conceptual exercises, and manipulatives to improve learning in the neuroanatomy classroom. Adv. Physiol. Educ., v. 32, n. 2, p. 152-156, 2008. Disponível em: <https://journals.physiology.org/doi/full/10.1152/advan.00103.2007>. Acesso em: 10 out. 2019. 
LENT, R. Cem bilhões de neurônios?; conceitos fundamentais de neurociência. 2. ed. São Paulo: Atheneu, 2010.

MACHADO, A. HAERTEL, L. M. Neuroanatomia funcional. 3. ed. São Paulo: Editora Atheneu, 2014.

MOTA, M. F.; MATA, F. R.; AVERSI-FERREIRA, T. A. Constructivist pedagogic method used in the teaching of human anatomy. Int. J. Morphol., v. 28, n. 2, p. 369-374, 2010. Disponível em:

<https://pdfs.semanticscholar.org/ada5/759d997502e8f4c7014dcd61c3c616de1814.pdf>. Acesso em: 12 mar. 2019.

PAULA, L. A. L. Jovens e novas formas de cognição: algumas reflexões sobre a escola. In: ARANHA, G.; SHOLLFRANCO, A. Caminhos da Neuroeducação. 2. ed. Rio de Janeiro: Ciências e Cognição, 2012.

RAMOS, K. S.; PEDROSO, A. C.; GUIMARÃES, G. F.; SANTOS, J. C. C.; LACERDA, P. S. D. Uma análise de caso acerca do ensino em morfologia na universidade do estado do Pará. In: XXIII CONGRESSO BRASILEIRO DE ANATOMIA, 2008, Belém. Anais... Belém: Universidade Federal do Pará, 2008.

RUIZ, C. R.; LIMA, N. B.; CUNHA, R. H.; NASCIMENTO, S. R. R. Investigation of the impact using neuroanatomy specimens prepared using new anatomical techniques on students' learning. J. Morphol. Sci., v. 31, n. 4, p. 210-213, 2014. Disponível em: <http://www.jms.periodikos.com.br/journal/jms/article/doi/10.4322/jms.063013>. Acesso em: 07 set. 2019.

SANTOS-LOBATO, B. L.; MAGALHÃES, A. B.; MOREIRA, D. G.; FARIAS, F. P.; PORTO, L. K.; PEREIRA, R. B.; CUSTÓDIO, S. S.; BRAGA, T. K. K. Neurophobia in Brazil: Detecting and Preventing a Global Issue. Rev. Bras. Educ. Med., v.42, n.1, p.121-128, 2018. . Disponível em: <http://www.scielo.br/scielo.php?script=sci_arttext\&pid=S010055022018000100121>. Acesso em: 08 out. 2019.

SILVA, M. S. L., MACEDO, M. T.; MACHADO, H. A.; SILVA, E. C.; NOVAES, C. C. B. S. Jogos educacionais como mediador de interação social e estímulo educacional: Olimpíadas de anatomia. In: CONNEPI, 2011, Natal. Anais... Natal: IFRN, 2011.

SILVA, M. S. L.; MACHADO, H. A.; BIAZUSSI, H. M. Produção de material didático alternativo para aula prática de anatomia humana. In: CONNEPI. 7., 2012, Palmas. Ciência, tecnologia e inovação: ações sustentáveis para o desenvolvimento regional. Disponível em:

<http://propi.ifto.edu.br/ocs/index.php/connepi/vii/paper/viewFile/4211/1560>. Acesso em: 14 mar. 2019.

SILVA, Y. A.; SILVA JÚNIOR, E. X.; SIVA, B. N.; RODRIGUES, G. P.; SOUSA, G. O.; NOVAES, W. A.; SHIOSAKI, R. K.; SILVA, T. F. A.; SCHWINGEL, P. A. Confecção de modelo neuroanatomico funcional como alternativa de ensino e aprendizagem para a disciplina de neuroanatomia. Revista Ibero-Americana de Estudos em Educação, v. 12, n. 3, p. 1674-1688, 2017. . Disponível em: <https://periodicos.fclar.unesp.br/iberoamericana/article/view/8502>. Acesso em: 21 abr. 2019.

SINGH, V.; KHARB, P. A paradigm shift from teaching to learning gross anatomy: meta-analysis of implications for instructuinal methods. J. Anatom. Soc. India, v. 62, p. 84-89, 2013. Disponível em: <http://medind.nic.in/jae/t13/i1/jaet13i1p84.pdf>. Acesso em: 12 mar. 2019

SHOLL-FRANCO, A.; VEQUE, T. Fazendo arte com o cérebro. Rio de Janeiro: Ciências e Cognição, 2013.

TAN, K.; CHIN, H. X.; YAU, C. W. L.; LIM, E. C. H.; SAMARASEKERA, D.; PONNAMPERUMA, G.; NCK, T. Evaluating a bedside tool for neuroanatomical localization with extended-matching questions. Anat. Sci. Educ., v.11, n. 3, p. 262-269, 2018. Disponível em: <https://www.academia.edu/34686668/Evaluating_a_bedside_tool_for_neuroanatomical_localization_with_extend ed-matching_questions>. Acesso em: 07 set. 2019.

\section{(c) EY}

Este trabalho está licenciado com uma Licença Creative Commons - Atribuição 4.0 Internacional. 